\title{
Comparison of Terra and Aqua MODIS VIS Bands On-orbit Response
}

\author{
X. Xiong \\ Sciences and Exploration Directorate, NASA/GSFC, Greenbelt, MD 20771 \\ Xiaoxiong.Xiong-1@nasa.gov \\ 301-614-5957
}

J. Sun, N. Che, T. Choi, and A. Angal

Science Systems and Applications, Inc., 10210 Greenbelt Road, Lanham, MD 20706

The Moderate Resolution Imaging Spectroradiometer (MODIS) has 36 spectral bands with a total of 490 detectors, covering spectral regions in the visible (VIS), near-infrared (NIR), short-wave infrared (SWIR), mid-wave infrared (MWIR), and long-wave infrared (LWIR). MODIS is a cross-track scanning radiometer which collects data using a rotating scan mirror (both sides) over a wide range of scan angles. The VIS, NIR, and SWIR bands (bands 1-19 and 26) make measurements of daytime surface reflected radiances, thus are referred to as the reflective solar bands (RSB). MODIS was built with a complete set of on-board calibrators, capable of providing radiometric, spatial, and spectral calibration and characterization during its entire mission. The RSB on-orbit calibration is primarily provided using a solar diffuser (SD) and a solar diffuser stability monitor (SDSM). The SD and SDSM calibration system is operated on a regular (weekly to tri-weekly) basis. The spectro-radiometric calibration assembly (SRCA) is another on-board calibrator that also provides RSB radiometric calibration support. For this purpose, the SRCA is operated in a radiometric mode on a monthly basis. A complete SRCA radiometric calibration is performed using different lamp configurations, or different radiance levels, to cover the range of RSB gain. Two additional SRCA modes with slightly different configurations are designed and operated for sensor on-orbit spectral and spatial characterization. In addition to its on-board calibrators, each MODIS makes monthly lunar observations to monitor RSB radiometric calibration stability. The MODIS lunar observations are made through its space view (SV) port at nearly the same lunar phase angles via spacecraft roll maneuvers. The SD, SRCA, and lunar measurements are made at different scan angles and data samples are collected for all spectral bands and detectors using both sides of the scan mirror.

Since launch, Terra and Aqua MODIS have operated successfully for more than 8 years and 6 years, respectively. Many SD/SDSM, SRCA, and lunar observations have been made by each instrument and used to derive RSB on-orbit calibration parameters, enabling corrections for sensor response changes and changes of the response versus scan angle (RVS). In general, the RSB calibration parameters are updated regularly into the MODIS Level 1B (L1B) code in support of continuous data processing for all MODIS science data products. This paper provides a brief description of MODIS RSB calibration methodologies and approaches, and summarizes on-orbit changes of their responses (gains), particularly for the VIS spectral bands. For both Terra and Aqua MODIS, the VIS band responses have shown larger on-orbit changes than other RSB 
bands (NIR and SWIR). Results presented in this paper will illustrate the response differences among detectors within a given VIS spectral band and that at different scan angles using SD, SRCA, and lunar observations. In addition, the differences between the two mirror sides and at different scan angles are presented for each sensor's mission lifetime. For Terra MODIS, a noticeable difference between responses from each side of the scan mirror has been observed in the VIS spectral bands. On the other hand, this mirror side dependent difference has been very small for Aqua MODIS VIS bands (less than $1 \%$ at $0.41 \mu \mathrm{m}$ and less than $0.5 \%$ at $0.48 \mu \mathrm{m}$ ). The RSB calibration is band, detector, and mirror side dependent. However, the RVS correction is only band averaged with no detector dependence in the current L1B algorithm. This study also tracks detector-to-detector response changes at different scan angles, providing valuable information for future improvements. 